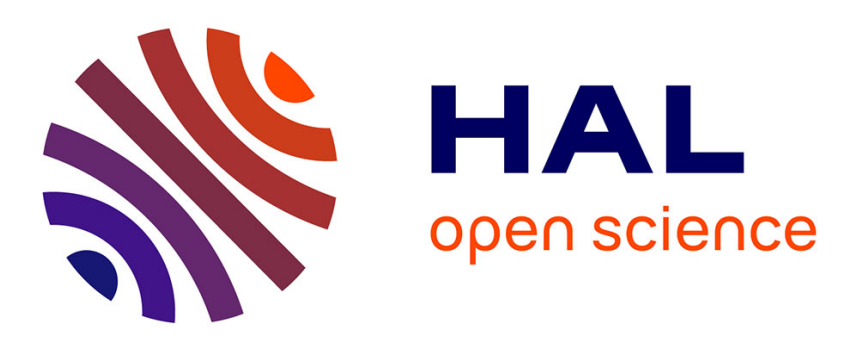

\title{
In situ synchrotron wide-angle X-ray diffraction investigation of fatigue cracks in natural rubber
}

Pierre Rublon, Bertrand Huneau, Nicolas Saintier, Stéphanie Beurrot, Adrien

Leygue, Erwan Verron, Cristian Mocuta, Dominique Thiaudière, Daniel

Berghezan

\section{To cite this version:}

Pierre Rublon, Bertrand Huneau, Nicolas Saintier, Stéphanie Beurrot, Adrien Leygue, et al.. In situ synchrotron wide-angle X-ray diffraction investigation of fatigue cracks in natural rubber. Journal of Synchrotron Radiation, 2013, 20, pp.105-109. 10.1107/S0909049512044457 . hal-01006961

\section{HAL Id: hal-01006961 https://hal.science/hal-01006961}

Submitted on 27 Sep 2017

HAL is a multi-disciplinary open access archive for the deposit and dissemination of scientific research documents, whether they are published or not. The documents may come from teaching and research institutions in France or abroad, or from public or private research centers.
L'archive ouverte pluridisciplinaire HAL, est destinée au dépôt et à la diffusion de documents scientifiques de niveau recherche, publiés ou non, émanant des établissements d'enseignement et de recherche français ou étrangers, des laboratoires publics ou privés. 


\title{
In situ synchrotron wide-angle X-ray diffraction investigation of fatigue cracks in natural rubber
}

\author{
Pierre Rublon, ${ }^{\mathrm{a}, \mathrm{b}}$ Bertrand Huneau, ${ }^{\mathrm{a} *}$ Nicolas Saintier, ${ }^{\mathrm{c}}$ Stéphanie Beurrot, ${ }^{\mathrm{a}}$ \\ Adrien Leygue, ${ }^{a}$ Erwan Verron, ${ }^{a}$ Cristian Mocuta, ${ }^{d}$ Dominique Thiaudière ${ }^{d}$ and \\ Daniel Berghezan ${ }^{b}$
}

'LUNAM Université, Ecole Centrale de Nantes, GeM, UMR CNRS 6183, BP 92101, 44321 Nantes

Cedex 3, France, ${ }^{\mathbf{b}}$ Michelin, Centre Technique de Ladoux, Clermont-Ferrand, France, ${ }^{\mathbf{c}}$ Arts et

Métiers ParisTech, Centre de Bordeaux-Talence, I2M, UMR CNRS 5295, Esplanade des Arts et

Métiers, 33405 Talence Cedex, France, and 'd Synchrotron Soleil, L'Orme des Merisiers, BP 48,

91192 Gif sur Yvette, France. E-mail: bertrand.huneau@ec-nantes.fr

Natural rubber exhibits remarkable mechanical fatigue properties usually attributed to strain-induced crystallization. To investigate this phenomenon, an original experimental set-up that couples synchrotron radiation with a homemade fatigue machine has been developed. Diffraction-pattern recording is synchronized with cyclic loading in order to obtain spatial distributions of crystallinity in the sample at prescribed times of the mechanical cycles. Then, real-time measurement of crystallinity is permitted during uninterrupted fatigue experiments. First results demonstrate the relevance of the method: the set-up is successfully used to measure the crystallinity distribution around a fatigue crack tip in a carbon black filled natural rubber for different loading conditions.

Keywords: strain-induced crystallization (SIC); WAXD; fatigue; crack; natural rubber.

\section{Introduction}

The study of fatigue crack propagation in elastomers is of major importance for the tyre industry to improve the service life of products. Vulcanized cis-1,4-polyisoprene (natural rubber; NR) is a key compound in tyres; it exhibits remarkable mechanical properties and more particularly a very good resistance to fatigue crack growth as compared with synthetic rubbers, especially for large strain (Lake, 1972; Young, 1986; Mars \& Fatemi, 2004; Papadopoulos et al., 2008). It is widely recognized that these excellent fatigue properties are related to the ability of NR to crystallize under strain (Lindley, 1973; Gent, 2002; Saintier et al., 2011). This property is usually referred to as strain-induced crystallization (SIC); the reader may consult Tanaka (2001) and Huneau (2011) for details on this subject.

Most of the works devoted to SIC of NR observed with realtime wide-angle X-ray diffraction (WAXD) measurements focus on uniaxial quasi-static tension-retraction tests (Toki et al., 2000; Trabelsi et al., 2003a; Rault et al., 2006; Chenal et al., 2007). Those studies highlight the relationship between the hysteresis of both the stress-strain curve and the diffracted intensity commonly used as a measure of strain-induced crystallinity. More recently, a few authors have investigated SIC under multiaxial loading conditions: Pannier et al. (2011) performed simultaneous uniaxial tension simple shear tests, whereas Beurrot et al. (2011) studied planar biaxiality. Besides, very few studies considered SIC under fatigue loading conditions. In the 1970s, Kawai (1975) successfully measured SIC in fatigue using a stroboscopic method in order to accumulate the weak diffracted intensity over hundreds of cycles. More recently, Rouvière et al. (2007) performed interrupted fatigue tests, WAXD acquisitions being performed at a fixed strain level during $45 \mathrm{~min}$; this method renders difficult the split between SIC due to fatigue and SIC due to maintained stretch during measurements. Beurrot-Borgarino et al. (2013) overcame this difficulty using synchrotron radiation to reduce the exposure time to $1 \mathrm{~s}$. Finally, it is noted that only two papers investigated the crystallinity in the neighbourhood of a crack tip: Lee \& Donovan (1987) measured the distribution of crystallinity along the propagation direction of a crack in both carbon black filled and unfilled NR, and Trabelsi et al. (2002) extended the WAXD measurements to the whole neighbourhood of the crack tip in unfilled NR. In both cases the measurements were performed at fixed stretch.

In this paper we present an original experimental set-up developed to measure in real-time the crystallinity around the crack tip in carbon black filled NR during fatigue. As shown in Fig. 1, the experiment consists of mapping the neighbourhood of the crack tip without stopping cyclic loading. This method allows us to determine the SIC distribution around a crack tip during uninterrupted fatigue tests, in order to distinguish SIC due to fatigue from SIC due to constant stretching, which differ: during cyclic experiments in which the sample is fully unloaded, crystallites melt completely (Albouy et al., 2012; Beurrot-Borgarino et al., 2013). We believe that the present 


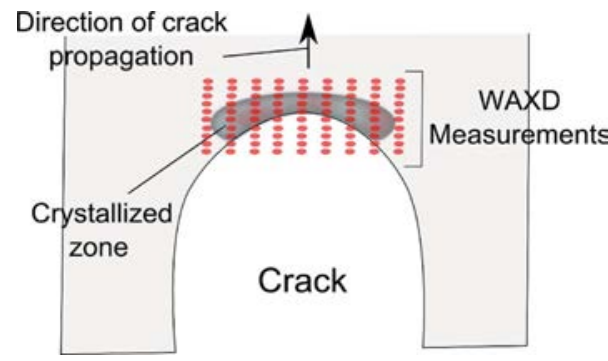

Figure 1

'Mapping' of WAXD measurements to investigate the crystallized zone at the crack tip. Red points represent the measurement points.

measurements help to explain the good properties of NR in fatigue and more precisely its good resistance to fatigue crack growth. This paper describes in detail the aforementioned original experimental method and a few results are proposed.

\section{Experimental set-up}

\subsection{Material and sample}

The material is a natural rubber filled with 50 p.h.r. (parts per hundred rubber) of N347 carbon black. Vulcanization is carried out with 1.6 p.h.r. of sulfur, where CBS acts as an accelerator. Each blend also contains $\mathrm{ZnO}$ and stearic acid. 6PPD is used as an antioxidant. The formulation is given in Table 1.

'Pure shear' specimens have been prepared and cured by Michelin; these types of specimens, also referred to as 'planar tension' specimens, are commonly used for fatigue crack growth measurements (Lake, 1972; Papadopoulos et al., 2008). They are $78 \mathrm{~mm}$ long, $13 \mathrm{~mm}$ high and $2 \mathrm{~mm}$ thick, as shown in Fig. 2.

\subsection{Testing machine}

Experiments were conducted with the homemade fatigue machine shown in Fig. 3. This apparatus was initially designed for a previous study where four electrical actuators were used to prescribe biaxial deformation to rubber cross-shaped samples (Beurrot et al., 2011). In the present case, only two actuators are used; they are supplied with sinusoidal waveforms to prescribe stretch to specimens. Their movements are synchronized in order to keep the centre of the specimen fixed

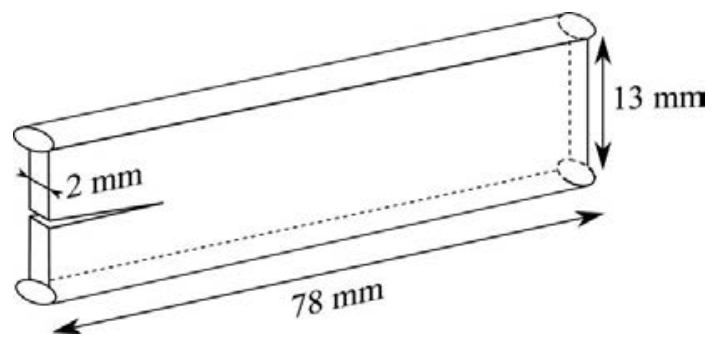

Figure 2

Pure shear specimen.
Table 1

Material formulation.

\begin{tabular}{ll}
\hline Ingredients & Content (p.h.r.) \\
\hline NR & 100 \\
Carbon black N347 & 50 \\
Stearic acid & 2 \\
Zinc oxide & 2.5 \\
CBS accelerator $\dagger$ & 1.6 \\
6PPD $\ddagger$ & 1.9 \\
Sulfur & 1.6 \\
\hline
\end{tabular}

$\dagger N$-Cyclohexyl-2-benzothiazole sulfonamide. $\ddagger N$-(1,3-dimethylbutyl)- $N$ '-phenyl- $p$ phenylenediamine.

during cyclic tests. Their loading capacity is $500 \mathrm{~N}$ and their stroke is $60 \mathrm{~mm}$. During all the tests the force is measured by means of a load cell (capacity: $1 \mathrm{kN}$ ). Special clamps have been designed for our pure shear samples. A particular fixture was designed to adapt this experimental set-up to the DiffAbs beamline at the French national synchrotron facility SOLEIL.

\subsection{Sample preparation}

Once clamped, the sample is first cycled for 300 cycles at a global stretch ratio of 1.92 and at a frequency of $2 \mathrm{~Hz}$ in order to lower the residual stretch due to viscous and Mullins effects. After this preconditioning stage the displacement of the clamps is adjusted in order to reach a force of $0 \mathrm{~N}$. A $20 \mathrm{~mm}$ long cut is then made in one side of the sample; as verified by finite element analysis, it is long enough to neglect the boundary influence on the stress field in the crack tip region. Next, we performed a preliminary cyclic test which permits to blunt the crack tip and to transform the cutter incision into a fatigue crack. A few hundreds of cycles are necessary to complete this transformation. It is also verified that the crack tip propagates by at least $1 \mathrm{~mm}$ from the cutter incision tip. This procedure is performed before each experiment at the same global stretch ratio as that used during measurements.

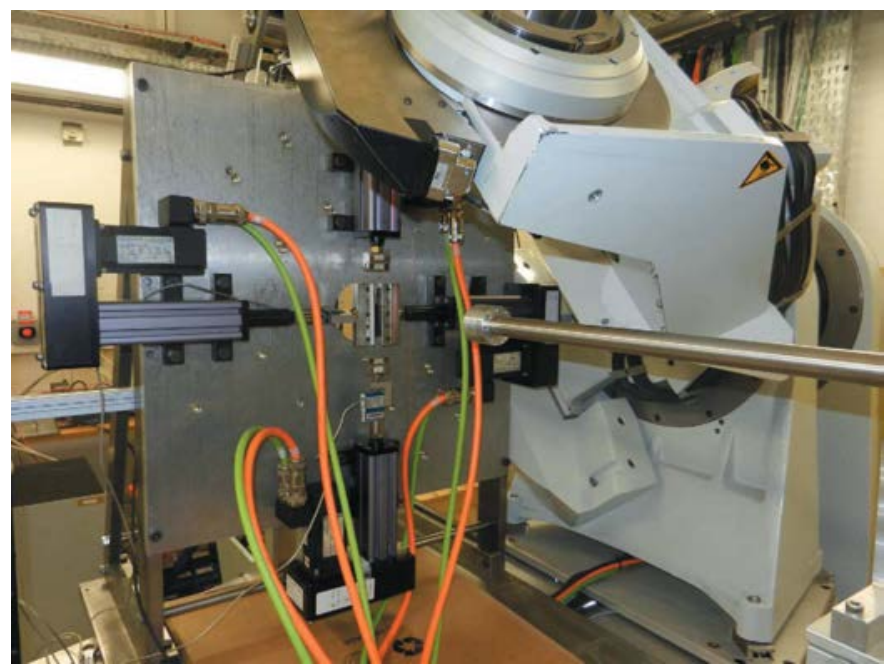

Figure 3

The fatigue machine on the DiffAbs diffractometer. 
Table 2

Dimensions and spacewidth of the map for each global stretch ratio.

\begin{tabular}{llllll}
\hline \multirow{2}{*}{$\begin{array}{l}\text { Global stretch } \\
\text { ratio }\end{array}$} & \multicolumn{2}{l}{ Global dimensions $(\mathrm{mm})$} & & \multicolumn{2}{l}{ Spacewidth $(\mathrm{mm})$} \\
& Height $(H)$ & Width $(W)$ & & Height $(H)$ & Width $(W)$ \\
\hline 1.46 & 1 & 2 & 0.111 & 0.25 \\
1.61 & 1.5 & 3.5 & 0.167 & 0.389 \\
1.77 & 2.5 & 5 & 0.278 & 0.625 \\
1.92 & 3 & 7 & 0.333 & 0.875 \\
\hline
\end{tabular}

\subsection{Beamline set-up}

In situ WAXD measurements were conducted at the DiffAbs beamline (Synchrotron SOLEIL). The wavelength was $0.1305 \mathrm{~nm}$ and the beam size was approximately $300 \mu \mathrm{m} \times$ $200 \mu \mathrm{m}$ (full width at half-maximum). The two-dimensional WAXD patterns are recorded using a MAR CCD X-ray detector (SX-165) with an exposure time of $1 \mathrm{~s}$. The intense transmitted beam is blocked by a $\mathrm{Pb}$ beamstop; a Si PIN photodiode placed in the centre of the beamstop measures the transmitted intensity through the sample in order to accurately estimate the thickness of the sample in the area exposed to the X-ray beam (simultaneously with WAXD acquisitions), and also to be able to locate the crack tip. We recall here that the tensile direction of the sample is perpendicular to the beam.

\section{Simultaneous mechanical loading and WAXD mapping}

\subsection{Position of the crack tip}

A particular procedure, described in the following, was developed to locate the fatigue crack tip after each loading sequence. The identified crack tip position was defined as the new reference point for the subsequent WAXD measurements. The procedure consists of slightly stretching the cracked sample, moving it with respect to the beam axis and simultaneously recording the transmitted intensity, using a photodiode, as an indicator of the presence of rubber in the beam. This procedure is performed in four steps, i.e. four different paths for the sample, as described in Fig. 4, and assumes that the crack tip is on an axis of symmetry located at equal distance from the two lips of the crack.

\subsection{Mapping of the crack tip neighbourhood during cyclic loading}

The approximate size of the crystallized area at the crack tip for each global stretch ratio has been determined thanks to several preliminary tests: the dimensions of the map, i.e. its height $H$ and width $W$, were chosen depending on the global stretch ratio; the corresponding values are given in Table 2. Then, the map is placed with respect to the reference point determined following the above-mentioned procedure: the reference point is at one-third of $H$ and at half of $W$, as depicted in the top part of Fig. 5.

After another 100 cycles in fatigue at a frequency of $2 \mathrm{~Hz}$, a fully automated method that couples the fatigue machine with

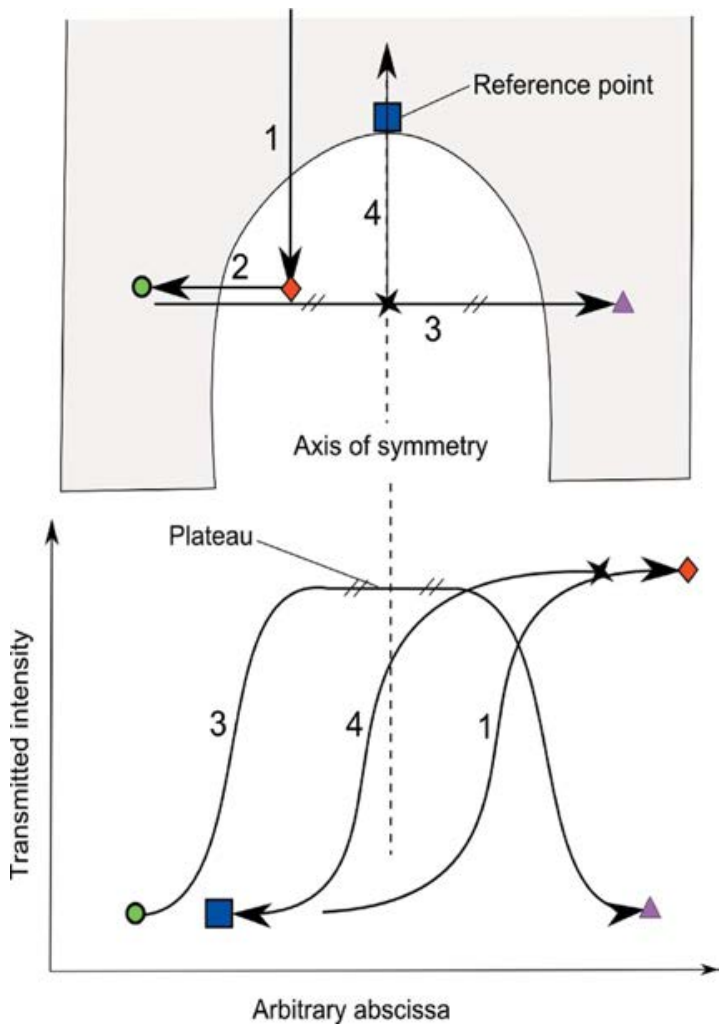

Figure 4

Determination of the crack tip position before WAXD measurements.
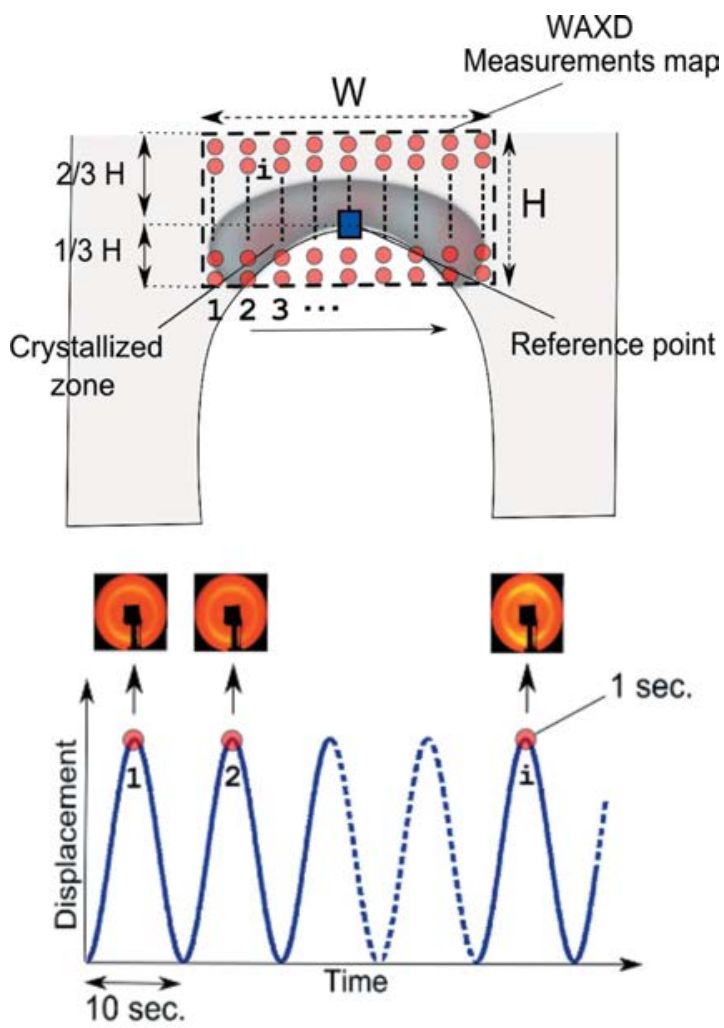

Figure 5

Top: mapping of the crack tip neighbourhood. Bottom: measurements during fatigue tests. The red points represent WAXD acquisitions. 
the beamline permits the acquisition of WAXD measurements around the crack tip, without interruption of the cyclic loading. It consists of recording a WAXD pattern at the maximum displacement of each cycle of the sinusoidal waveform, and moving the sample with respect to the beam between two cycles to reach the next point of measurement in the prescribed map, as described in Fig. 5. It is noteworthy that, to move the sample, the whole diffractometer of the beamline, on which the fatigue machine is placed, is translated. The Si PIN photodiode is moved automatically to compensate this translation. In this way it is kept centred with respect to the beam. In order to permit the diffractometer translation between two cycles, and to allow the $1 \mathrm{~s}$ recording at each maximum, it is necessary to impose a lower loading frequency, i.e. $0.1 \mathrm{~Hz}$. As SIC is a rapid process (Albouy et al., 2012; Candau et al., 2012; Bruning et al., 2012), we assume that the results observed at $0.1 \mathrm{~Hz}$ would be similar to those observed at $2 \mathrm{~Hz}$, even if the crystallized zone is probably larger at $0.1 \mathrm{~Hz}$ because of the time-dependency of SIC. Considering that the exposure time is $1 \mathrm{~s}$, a trigger is sent by the stretching machine to the beamline $0.5 \mathrm{~s}$ before each maximum displacement. Because of the very small variation of the global displacement during this $1 \mathrm{~s}$ acquisition (less than $2.5 \%$ ), the WAXD pattern is relevant. At the end of this recording the diffractometer is translated within $10 \mathrm{~s}$ between two cycles, and waits for the next trigger from the fatigue machine to acquire the next WAXD pattern defined in the map. Each map contains 90 diffraction patterns $(9 \times 10$ points $)$. It was considered that during those 90 cycles the growth of the crack is negligible compared with the map size in the fatigue crack propagation direction; this assumption has been confirmed $a$ posteriori with the measurement of the crack length after each loading sequence and with fatigue crack growth rate measurements performed at $0.1 \mathrm{~Hz}$. One notes that for a constant number of measurement points the spacewidth (distance between two measurement points) changes with the map dimensions (see Table 2).

\section{Analysis of WAXD patterns}

\subsection{Method}

For each pattern the diffracted intensity is first corrected by taking into account the change in both the sample thickness due to extension and the intensity of the incident beam, following the method of Ran et al. (2001). An example of a WAXD pattern is shown in Fig. 6. Secondly, we determine the total diffracted intensity along one radius of the pattern $I_{\text {total }}(2 \theta)$ at the azimuthal angles corresponding to the (200) and (120) reflection arcs. Third, these data are fitted by a series of Pearson VII functions, similarly as in Trabelsi et al. (2003a), Chenal et al. (2007) and Saintier et al. (2011). It should be noted that a linear baseline is suppressed before the aforementioned deconvolution. This suppression makes the deconvolution easier, and permits the fluctuation in total diffracted intensity between two recordings to be overcome. An example of fitted data is presented in Fig. 7.



Figure 6

An example of a WAXD pattern.

For low crystallinity levels the (120) Bragg reflection is difficult to deconvolute from the amorphous peak. Furthermore, the deconvolution of the (200) peak leads to an excessive broadening of the associated Pearson function whose area gives an overestimated value of the index of crystallinity. A way to overcome this difficulty is to use only the height of the (200) peak rather than the integrated area, assuming consequently a constant crystallite size with stretch. Since the present study focuses on only one filled natural rubber, this assumption is thought to be consistent with the literature: for example, Trabelsi et al. (2003b) report an almost constant crystallite size with stretch ratio in a filled NR.

Our index of crystallinity is finally calculated as follows,

$$
\chi=I_{200} /\left(I_{200}+I_{\text {amorphous }}\right),
$$

where $I_{200}$ is the maximum intensity of the (200) Bragg reflection and $I_{\text {amorphous }}$ is the intensity of the amorphous peak. The authors are aware that this index is coarse. Nevertheless, it allows the crystallinity distribution in the neighbourhood of the crack tip to be rapidly estimated and is sufficient for the present purpose.

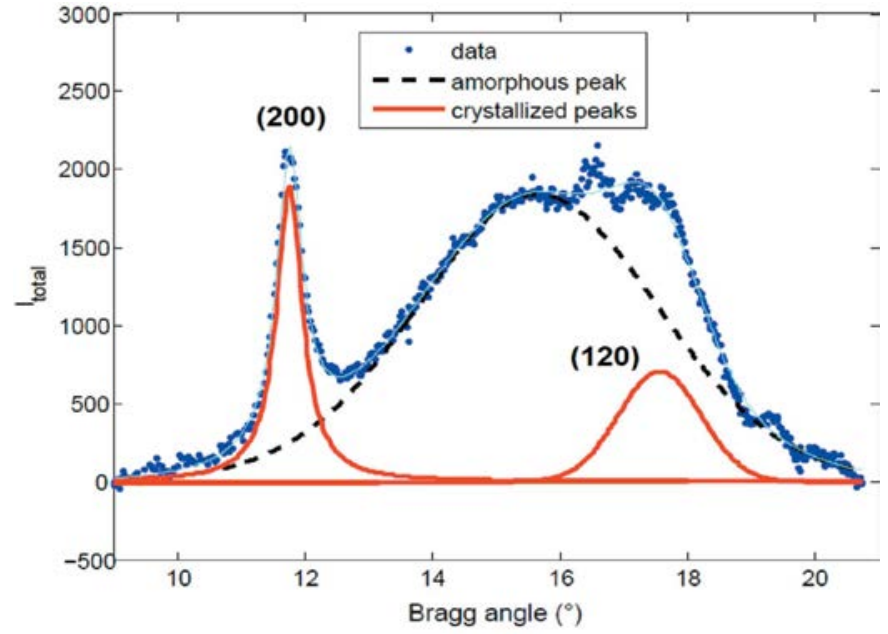

Figure 7

Deconvolution and fitting of a pattern. 


\subsection{First results}

Once the mapping is recorded, a post-processing analysis leads to the plot of 'iso-crystallinity' curves that gives the distribution of SIC around the crack tip. Fig. 8 presents these iso-crystallinity curves for four global stretch ratios, i.e. $\lambda=$ $1.46, \lambda=1.61, \lambda=1.77$ and $\lambda=1.92$. In these examples the minimal value of the crystallinity index is 0.1 . Below this value we were unable to accurately deconvolute both crystallized and amorphous parts because of the very small size of the (200) peak. The maximum value is equal to 0.4 . It is worth noting that, because of the large size of the beam, the quantitative evaluation of crystallinity in the region very close to the crack tip is difficult and less accurate. These results demonstrate that the global stretch ratio has a noteworthy influence on the size of the crystallized zone at the crack tip: as an example, the dimensions of the crystallized area are doubled in both directions between $\lambda=1.61$ and $\lambda=1.92$. Even if this result can be considered as intuitive, it had never been measured under fatigue loading conditions. Furthermore, one can note that the size of the crystallized zone measured here appears to be much larger than that reported by Trabelsi et al. (2002). This difference is mainly due to the use of different materials (unfilled NR versus filled NR), but also of different specimen geometries (simple extension versus pure shear specimens). Concerning the impact of fillers, Lee \& Donovan (1987) observed dimensions of the crystallized zone on carbon black filled samples (40 p.h.r. of carbon black),

(a)

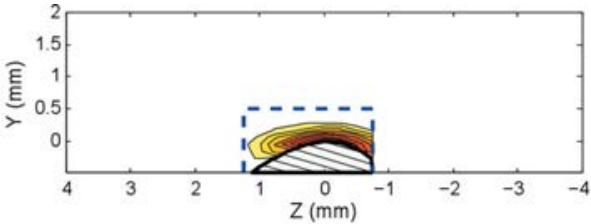

(b)

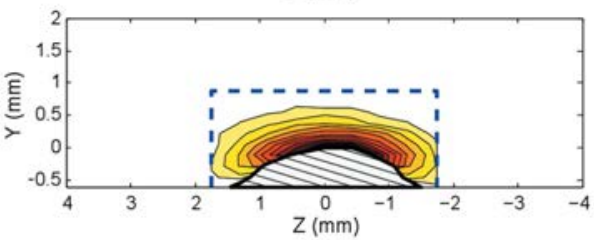

(c)

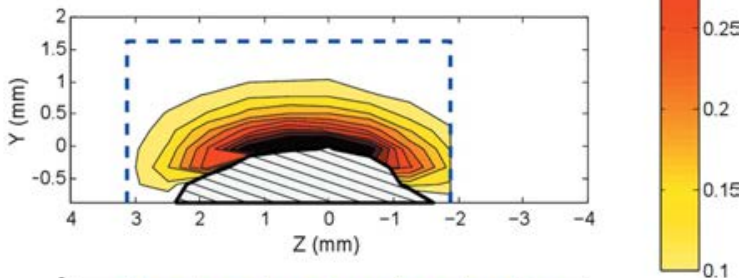

(d)

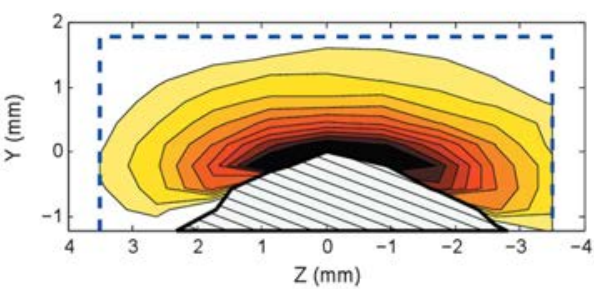

Figure 8

Index of crystallinity around a fatigue crack tip for different global stretch ratios: (a) 1.46, (b) 1.61, (c) 1.77 and (d) 1.92. Dotted lines show the measurement maps and the hatched zones represent the crack. which are in good agreement with the present results (about $1-2 \mathrm{~mm}$ in the axis of the crack).

\section{Conclusion}

The original experimental set-up presented here consists of the association of a homemade fatigue machine and synchrotron radiation. It permits the measurement of crystallinity in elastomers during fatigue tests without stopping the mechanical loading. With this device it is possible to measure crystallinity at prescribed times and positions. In the present study it has been applied to fatigue cracks in pure shear carbon black filled natural rubber samples. It leads to the twodimensional distribution of SIC in the neigbourhood of the crack tip. Further investigations are in progress to evaluate the influence of filler content as well as that of loading conditions.

All the reported diffraction measurements were performed on the DiffAbs beamline at Synchrotron SOLEIL, Gif-surYvette, France (proposal 20110204).

\section{References}

Albouy, P.-A., Guillier, G., Petermann, D., Vieyres, A., Sanseau, O. \& Sotta, P. (2012). Polymer, 53, 3313-3324.

Beurrot-Borgarino, S., Huneau, B., Verron, E. \& Rublon, P. (2013). Int. J. Fatigue, 47, 1-7.

Beurrot, S., Huneau, B. \& Verron, E. (2011). Constitutive Models for Rubber VII, edited by S. Jerrams and N. Murphy, pp. 23-28. London: Taylor and Francis.

Bruning, K., Schneider, K., Roth, S. V. \& Heinrich, G. (2012). Macromolecules, 45, 7914-7919.

Candau, N., Chazeau, L., Chenal, J.-M., Gauthier, C., Ferreira, J., Munch, E. \& Rochas, C. (2012). Polymer, 53, 2540-2543.

Chenal, J.-M., Gauthier, C., Chazeau, L., Guy, L. \& Bomal, Y. (2007). Polymer, 48, 6893-6901.

Gent, A. (2002). Rubber Chem. Technol. 75, 923-934.

Huneau, B. (2011). Rubber Chem. Technol. 84, 425-452.

Kawai, H. (1975). Rheol. Acta, 14, 27-47.

Lake, G. (1972). Rubber Chem. Technol. 45, 309-328.

Lee, D. \& Donovan, J. (1987). Rubber Chem. Technol. 60, 910-923.

Lindley, P. (1973). Int. J. Fract. 9, 449-462.

Mars, W. V. \& Fatemi, A. (2004). Rubber Chem. Technol. 77, 419-423.

Pannier, Y., Proudhon, H., Mocuta, C., Thiaudière, D. \& Cantournet, S. (2011). J. Synchrotron Rad. 18, 907-911.

Papadopoulos, I. C., Thomas, A. G. \& Busfield, J. J. C. (2008). Polymer, 109, 1900-1910.

Ran, S., Fang, D., Zong, X., Hsiao, B. S., Chu, B. \& Cunniff, P. M. (2001). Polymer, 42, 1601-1612.

Rault, J., Marchal, J., Judeinstein, P. \& Albouy, P. A. (2006). Eur. Phys. J. E, 21, 243-261.

Rouvière, J.-Y., Bennani, A., Pachoutinsky, D., Besson, J. \& Cantournet, S. (2007). Constitutive Models for Rubber V, edited by A. Boukamel, L. Laiarinandrasana, S. Méo and E. Verron, pp. 323-326. London: Taylor and Francis.

Saintier, N., Cailletaud, G. \& Piques, R. (2011). Mater. Sci. Eng. A, 528, 1078-1086.

Tanaka, Y. (2001). Rubber Chem. Technol. 74, 355-375.

Toki, S., Fujimaki, T. \& Okuyama, M. (2000). Polymer, 41, 5423-5429.

Trabelsi, S., Albouy, P.-A. \& Rault, J. (2002). Macromolecules, 35, 10054-10061.

Trabelsi, S., Albouy, P.-A. \& Rault, J. (2003a). Macromolecules, 36, 7624-7639.

Trabelsi, S., Albouy, P.-A. \& Rault, J. (2003b). Macromolecules, 36, 9093-9099.

Young, D. (1986). Rubber Chem. Technol. 59, 809-825. 